Revista Brasil. Bot., V.25, n.1, p.11-17, mar. 2002

\title{
Aves como potenciais dispersoras de sementes de Ocotea pulchella Mart. (Lauraceae) numa área de vegetação de cerrado do sudeste brasileiro
}

\author{
MERCIVAL R. FRANCISCO ${ }^{1,3}$ e MAURO GALETTI ${ }^{2}$
}

(recebido: 2 de agosto de 2000; aceito: 26 de setembro de 2001)

\begin{abstract}
Birds as potential seed dispersers of Ocotea pulchella Mart. (Lauraceae) in a cerrado area from southeastern Brazil). Studies on frugivory and seed dispersal are extremely important for management plans, but few studies have been developed in the Brazilian cerrado. In this paper we describe the frugivory and seed dispersal of Ocotea pulchella Mart. (Lauraceae) by birds in a cerrado area in São Paulo state (21 $1^{\circ} 58^{\prime} \mathrm{S}, 47^{\circ} 52^{\prime} \mathrm{W}$ ), southeastern Brazil. In 72 hours of focal observations, from August to October 1999, we recorded 411 visits of 13 different bird species consuming the fruits of $O$. pulchella. The main potential seed dispersers were the Pale-breasted Thrush, Turdus leucomelas (Muscicapidae), the Chalk-browed Mockingbird, Mimus saturninus (Mimidae), the Great Kiskadee, Pitangus sulphuratus (Tyrannidae) and the Elaenias, Elaenia spp. (Tyrannidae). We did not find significant difference in the frequency of visits at different hour intervals throughout the day when all of the species were pooled together and when the species were individually considered. The bird species did not differ in the time they spent foraging on the plants. The number of fruits consumed in each visit differed among species and there was positive correlation between avian body mass and mean number of consumed fruits in these visits. The time spent on plants was positively correlated to the number of consumed fruits by Elaenia spp., Brown-crested Flycatcher, Myiarchus tyrannulus (Tyrannidae) and the Rufous-bellied Thrush, Turdus rufiventris (Muscicapidae). Although specialist frugivorous birds have not been observed consuming $O$. pulchella, generalist species were potential seed dispersers, presenting high frequency of visits, high fruit consume and shortened visit periods.
\end{abstract}

RESUMO - (Aves como potenciais dispersoras de sementes de Ocotea pulchella Mart. (Lauraceae) numa área de vegetação de cerrado do sudeste brasileiro). Embora os estudos de frugivoria e dispersão de sementes sejam importantes para a realização de planos de manejo e recuperação de ambientes degradados, pouco tem sido pesquisado sobre o assunto no cerrado brasileiro. Neste trabalho são apresentados aspectos da frugivoria e dispersão das sementes de Ocotea pulchella Mart. (Lauraceae) por aves. Treze espécies de aves foram registradas consumindo os frutos de $O$. pulchella em 72 horas de observação focal, entre os

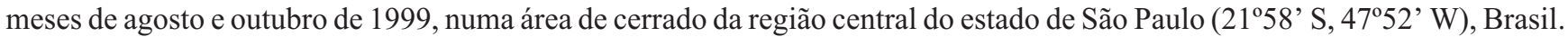
As principais espécies potencialmente dispersoras foram o sabiá-pardão, Turdus leucomelas (Muscicapidae), o sabiá-do-campo, Mimus saturninus (Mimidae), o bem-te-vi, Pitangus sulphuratus (Tyrannidae) e as guaracavas, Elaenia spp. (Tyrannidae). Não foram encontradas variações significativas no número de visitas nos diferentes intervalos de hora quando todas as espécies foram analisadas em conjunto e quando cada espécie foi analisada separadamente. Não houve variação significativa também no tempo de permanência sobre as plantas entre as diferentes espécies de aves. A taxa de consumo diferiu significativamente entre elas, tendo sido encontrada correlação positiva significativa entre o peso das diferentes espécies e o número médio de frutos consumidos por visita. O tempo de permanência sobre a planta e o número de frutos consumidos foram positivamente correlacionados para Elaenia spp., para a maria-cavaleira, Myiarchus tyrannulus (Tyrannidae) e para o sabiá-laranjeira, Turdus rufiventris (Muscicapidae). Embora espécies primariamente frugívoras não tenham sido observadas consumindo os frutos de O. pulchella, as espécies oportunistas pareceram favorecer a eficiência da dispersão, mantendo altas freqüências de visitas, altas taxas de consumo e permanecendo por curtos períodos de tempo sobre as plantas.

Key words - Seed dispersal, Ocotea pulchella, cerrado, frugivory, ornitochory

\section{Introdução}

A dispersão das sementes é um importante passo do ciclo reprodutivo da maioria das plantas (Herrera

\footnotetext{
1. Universidade Federal de São Carlos, Programa de Pós-Graduação em Ecologia e Recursos Naturais, Departamento de Genética e Evolução, Laboratório de Citogenética, Rod. Washington Luis, km 235, Caixa Postal 676, 13565-905 São Carlos, SP, Brasil.

2. Universidade Estadual Paulista, Departamento de Ecologia, Plant Phenology and Seed Dispersal Research Group, 13506-900 Rio Claro, SP, Brasil.

3. Autor para correspondência: pmrf@iris.ufscar.br
}

et al. 1994, Wenny \& Levey 1998). A dispersão por animais frugívoros constitui um processo simbiótico no qual as plantas têm suas sementes dispersas e os dispersores, em troca, recebem um retorno nutricional na forma de um pericarpo carnoso (Van der Pijl 1982, Coates-Estrada \& Estrada 1988).

Dentre algumas das hipóteses que tentam explicar as vantagens da dispersão de sementes estão o escape das altas taxas de mortalidade de sementes e plântulas sob e próximo às plantas adultas (hipótese do escape), a colonização de novos ambientes (hipótese da coloniza- 
ção) e a dispersão para microhabitats favoráveis (hipótese da dispersão direta) (Howe \& Smallwood 1982), hipóteses essas que não são excludentes (Wenny \& Levey 1998).

Estima-se que 50 a $90 \%$ das espécies de árvores encontradas nas florestas tropicais produzam frutos cujas sementes são dispersas por animais (Howe \& Smallwood 1982). Aproximadamente um terço das espécies de aves de muitas destas florestas são frugívoras, contribuindo com grande parte deste processo de dispersão (Snow 1981). Esta porcentagem se tornaria ainda maior se fossem consideradas as aves que se alimentam de frutos apenas ocasionalmente (Blake et al. 1990).

No entanto, diversos estudos têm demonstrado que as espécies de animais não apresentam a mesma eficiência como dispersoras (Howe \& Estabrook 1977, Snow 1981, Wheelwright \& Orians 1982, Levey 1987, Schupp 1993), sendo que alguns dos fatores mais importantes que podem influenciar na eficiência deste processo pelos diferentes agentes são o número de visitas à planta, o número de sementes dispersadas por visita, a qualidade do tratamento dado à semente, bem como a qualidade da deposição destas sementes (Schupp 1993).

Grande parte dos estudos de dispersão de sementes por aves tem se baseado na observação da utilização de uma única espécie de planta por várias espécies de aves (Howe 1977, Foster 1987, Motta-Junior \& Lombardi 1990, Herrera et al. 1994, Traveset 1994, Galetti \& Stotz 1996, Pizo 1997, Wenny \& Levey 1998). Estas aves, freqüentemente representam uma ampla variedade taxonômica, exibindo grande diversidade trófica e morfológica (Foster 1987, Galetti \& Pizo 1996), contribuindo muitas vezes de maneiras diferenciadas para o processo de dispersão.

Ocotea pulchella Mart. (Lauraceae) é uma planta semidecídua que chega a atingir $30 \mathrm{~m}$ de altura e $80 \mathrm{~cm}$ de diâmetro de caule, sendo bastante comum em ambientes de cerrado, matas e restingas. Distribui-se no Brasil pelos estados de Espírito Santo, Minas Gerais e Mato Grosso do Sul, até o Rio Grande do Sul (Lorenzi 1998). A frutificação é abundante e os frutos são elipsóides de coloração púrpura quando maduros. Um relato prévio do consumo de seus frutos por aves foi feito por Lorenzi (1998), embora não existam estudos específicos de frugivoria.

Diante disso, os objetivos deste trabalho foram determinar quais espécies de aves são consumidoras dos frutos de Ocotea pulchella numa área de transição entre vegetação de mata galeria e cerrado da região central do estado de São Paulo, Brasil, e através da análise dos números de frutos consumidos, tempos de permanência sobre as plantas e comportamentos de manipulação dos frutos, inferir sobre a potencialidade destas espécies para a dispersão das sementes.

\section{Material e métodos}

Este estudo foi realizado nas áreas não urbanizadas pertencentes ao campus da Universidade Federal de São Carlos (21 $58^{\prime}$ S, 47 $52^{\circ}$ ' W), região central do estado de São Paulo, Brasil. A área conta com 124,68 ha de vegetação de cerrado, 3,60 ha de matas ciliares, 93,84 ha de eucaliptais com sub-bosque de cerrado e 222,73 ha de silvicultura de Eucalyptus, além de 83,67 ha compostos por represas, trilhas e campos alterados. O local é alterado, sofrendo ações antrópicas esporádicas com atividades de retirada de Eucalyptus e queimadas ocasionais. O clima da região é tropical com verão úmido e inverno seco, sendo os mínimos pluviométricos atingidos geralmente entre os meses de abril e setembro (Paese 1997).

Levantamentos feitos por Motta-Junior \& Vasconcellos (1996) registraram para esta área um total de 214 espécies de aves. Observações mais recentes acrescentaram nove novas espécies à esta lista. Do total de espécies, 33\% consomem frutos pelo menos esporadicamente, o que sugere que as aves sejam responsáveis por uma parcela significativa da dispersão das sementes neste local.

Foram observados quatro indivíduos de Ocotea pulchella Mart. numa área de transição entre cerrado e mata galeria, os quais apresentaram no início do mês de agosto de 1999 cerca de $8.000,12.000,13.200$ e 19.200 frutos. A estimativa do número de frutos foi feita através de contagem direta, utilizando-se a média aproximada de três contagens. As plantas observadas estavam distanciadas por pelo menos $30 \mathrm{~m}$ de outras plantas da mesma espécie que também estivessem frutificando.

Os dados foram coletados em seis períodos completos de 12 horas de observação focal, das 6:00 às 18:00 h, seguindose a metodologia proposta por Pizo (1997), na qual durante as sessões de observação são registradas as espécies de aves consumidoras dos frutos, o número de visitas de cada espécie, incluindo-se as visitas em que não há consumo e o comportamento de manipulação dos frutos. Foi também anotado o horário exato de cada visita. Para isto, foram feitas 13 excursões ao campo entre os meses de agosto e outubro de 1999. Foram realizados dois períodos de 12 horas de observação para cada uma das plantas com 12.000 e 13.200 frutos e um único período para as demais. As observações foram feitas em barracas localizadas a $15 \mathrm{~m}$ de distância das plantas, com binóculos $7 \times 35 \mathrm{~mm}$.

Os números de frutos consumidos e os tempos de permanência sobre as plantas foram determinados a partir dos dados de observações completas, onde os animais puderam ser observados sem serem perdidos de vista durante todo o período da visita. A retirada de pedaços da polpa sem a 
remoção do fruto não foi considerada como consumo. A porcentagem de consumo para cada espécie foi calculada a partir da multiplicação do número médio de frutos consumidos por visita pelo número total de visitas, dividido pela somatória dos valores obtidos para todas as espécies e multiplicado por 100.

Para as análises de correlação foi aplicado o coeficiente de correlação de Spearman e para a verificação destas correlações foram utilizados os valores médios de peso das aves e dos números de frutos consumidos para cada espécie. Devido ao fato de os dados de tempo de permanência sobre as plantas, número de frutos consumidos e número de visitas por intervalo de hora terem apresentado coeficientes de variação altos (maiores de 15\%) para algumas espécies, foram aplicados testes não paramétricos para as demais análises. Os procedimentos estatísticos seguiram Sokal \& Rohlf(1981). As espécies com três ou menos observações completas não foram incluídas nas análises.

Por constituir-se num fator negativo para a dispersão, foram registrados os encontros agonísticos inter e intraespecíficos. Para isso, as investidas diretas, mesmo sem contatos físicos, também foram consideradas. A classificação das espécies quanto à dieta seguiu Willis (1979) e MottaJunior (1990) e a classificação quanto ao status migratório se baseou em Motta-Junior \& Vasconcellos (1996) e em observações pessoais. Os dados referentes aos pesos das aves foram obtidos de Marini et al. (1997) e Sick (1997).

As medidas dos frutos e sementes, as quais influenciam diretamente no comportamento de manipulação dos frutos pelas aves (Wheelwright 1985), foram tomadas com paquímetros de metal com $0,1 \mathrm{~mm}$ de precisão. Os pesos foram obtidos com balança elétrica (Micronal) de $0,01 \mathrm{~g}$.

\section{Resultados}

Frutos maduros de Ocotea pulchella foram encontrados entre os meses de agosto e outubro de 1999. Os frutos $(\mathrm{n}=30)$ mediram (média \pm desvio padrão) $8,14 \pm 0,52 \mathrm{~mm}$ de comprimento, $5,68 \pm 0,46 \mathrm{~mm}$ de largura e pesaram $0,15 \pm 0,034 \mathrm{~g}$. A proporção do peso das sementes em relação ao peso total dos diásporos foi $0,37 \pm 0,08$, medindo $7,25 \pm 0,83 \mathrm{~mm}$ de comprimento, $4,18 \pm 0,56 \mathrm{~mm}$ de largura e pesando $0,057 \pm 0,021 \mathrm{~g}$.

Durante 72 horas de observações foram registradas 13 espécies de aves pertencentes a quatro famílias diferentes consumindo frutos de $O$. pulchella (tabela 1), totalizando 411 visitas, com um número médio de 5,8 \pm $5,7$ (mediana $=4,0)$ visitas por hora. Todas as espécies

Tabela 1. Espécies de aves visitando Ocotea pulchella em 72 horas de observação. Nomenclatura sistemática de acordo com Sick (1997).

\begin{tabular}{|c|c|c|c|c|c|c|}
\hline Espécies & $\begin{array}{l}\text { Número } \\
\text { de visitas }\end{array}$ & Dieta $^{a}$ & Status ${ }^{\mathrm{b}}$ & N.c & Consumo $^{\mathrm{d}}$ & 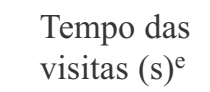 \\
\hline \multicolumn{7}{|l|}{ Tyrannidae } \\
\hline Elaenia spp. (guaracavas) & 95 & ONI & $\mathrm{R}$ & 10 & $1,8 \pm 0,9$ & $84,5 \pm 89,4$ \\
\hline Myiarchus tyrannulus (maria-cavaleira) & 18 & INS & $\mathrm{R}$ & 9 & $1,3 \pm 1,2$ & $75,2 \pm 60,9$ \\
\hline Pitangus sulphuratus (bem-te-vi) & 53 & ONI & $\mathrm{R}$ & 11 & $4,6 \pm 3,8$ & $108,3 \pm 70,7$ \\
\hline Myiozetetes similis (bem-te-vi-pequeno) & 9 & ONI & $\mathrm{R}$ & 4 & $2,2 \pm 2,6$ & $197 \pm 146$ \\
\hline Myiodynastes maculatus (bem-te-vi-rajado) & 3 & ONI & M & 2 & $5 / 1$ & $253 / 110$ \\
\hline Empidonomus varius (peitica) & 1 & ONI & M & 1 & 2 & 56 \\
\hline Tyrannus savana (tesourinha) & 36 & INS & $\mathrm{M}$ & 10 & $3,9 \pm 2,6$ & $425,6 \pm 668,9$ \\
\hline Tyrannus melancholicus (siriri) & 26 & INS & $\mathrm{R}$ & 8 & $2,0 \pm 3,5$ & $176,7 \pm 182,9$ \\
\hline \multicolumn{7}{|l|}{ Muscicapidae } \\
\hline Turdus rufiventris (sabiá-laranjeira) & 16 & ONI & $\mathrm{R}$ & 5 & $8,6 \pm 6,3$ & $603,2 \pm 629,2$ \\
\hline Turdus leucomelas (sabiá-pardão) & 66 & ONI & $\mathrm{R}$ & 10 & $4,5 \pm 3,0$ & $143,6 \pm 103,8$ \\
\hline Turdus amaurochalinus (sabiá-poca) & 19 & ONI & $\mathrm{R}$ & 7 & $3,0 \pm 1,5$ & $133,1 \pm 82,4$ \\
\hline \multicolumn{7}{|l|}{ Mimidae } \\
\hline Mimus saturninus (sabiá-do-campo) & 53 & ONI & $\mathrm{R}$ & 10 & $5,5 \pm 4,4$ & $224,4 \pm 131,6$ \\
\hline \multicolumn{7}{|l|}{ Emberizidae } \\
\hline Schistochlamys ruficapillus (bico-de-veludo) & 16 & ONI & $\mathrm{R}$ & 11 & $2,6 \pm 2,1$ & $181,4 \pm 146,3$ \\
\hline
\end{tabular}

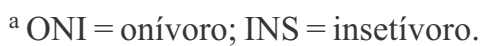

${ }^{\mathrm{b}} \mathrm{R}=$ residente; $\mathrm{M}=$ migratório.

${ }^{c}$ Número de observações com medidas completas do tempo de duração das visitas e do total de frutos consumidos.

${ }^{\mathrm{d}}$ Número médio de frutos consumidos por visita (média \pm desv. padrão).

e Tempo médio de permanência sobre as plantas por visita (média \pm desv. padrão). 
foram consideradas como potenciais dispersoras das sementes por engolirem os frutos inteiros (tabela 2), possibilitando às sementes serem regurgitadas em locais afastados das plantas parentais.

Tabela 2. Comportamentos de manipulação dos frutos de Ocotea pulchella por aves.

\begin{tabular}{lccc}
\hline \multirow{2}{*}{ Espécies } & \multicolumn{3}{c}{$\begin{array}{c}\text { Comportamento de } \\
\text { manipulação dos frutos }\end{array}$} \\
\cline { 2 - 4 } & EFI & CPS & MFP \\
\hline Elaenia spp. & 13 & - & 5 \\
Myiarchus tyrannulus & 11 & - & 1 \\
Pitangus sulphuratus & 50 & - & 1 \\
Myiozetetes similis & 9 & - & - \\
Myiodynastes maculatus & 6 & - & - \\
Empidonomus varius & 2 & - & - \\
Tyrannus savana & 36 & - & 3 \\
Tyrannus melancholicus & 16 & - & 1 \\
Turdus rufiventris & 38 & - & 5 \\
Turdus leucomelas & 54 & - & 1 \\
Turdus amaurochalinus & 21 & - & - \\
Mimus saturninus & 53 & - & 2 \\
Schystochlamys ruficapillus & 25 & 1 & 3 \\
\hline
\end{tabular}

${ }^{\text {a }} \mathrm{EFI}=$ Engole o fruto inteiro; CPS $=$ Consome pedaços da polpa sem remover o fruto; MFP = Mandibula o fruto deixando a semente ou o fruto todo cair sob a planta.

Algumas espécies de aves do gênero Elaenia são extremamente semelhantes, sendo a vocalização a melhor maneira para identificá-las no campo (Sick 1997). Poucas vezes estas aves foram observadas vocalizando durante as visitas às plantas, o que impossibilitou a distinção entre as três espécies presentes na área de estudo no período em que este trabalho foi realizado, $E$. chiriquensis, E. obscura e E. flavogaster.

As maiores porcentagens de consumo foram apresentadas por Turdus leucomelas (20,1\%), seguido por Mimus saturninus (19,7\%), Pitangus sulphuratus (16,6\%), Elaenia spp. (11,6\%), Tyrannus savana (9,5\%) e por Turdus rufiventris $(9,3 \%)$. As demais espécies foram responsáveis por $13,2 \%$ do consumo.

As espécies onívoras foram responsáveis por $85,4 \%$ do total de frutos consumidos, sendo seguidas pelas insetívoras, com 14,6\%. Espécies exclusivamente frugívoras, bem como predadoras de sementes não foram registradas. Três das espécies visitantes, Myiodinastes maculatus, Empidonomus varius e T. savana foram consideradas como migratórias (tabela 1), tendo sido juntas responsáveis por $9,5 \%$ do consumo dos diásporos.
O total de horas de observação acumulado, aparentemente amostrou todas as espécies de aves consumidoras de $O$. pulchella na área em estudo após 39 horas de observação (figura 1).

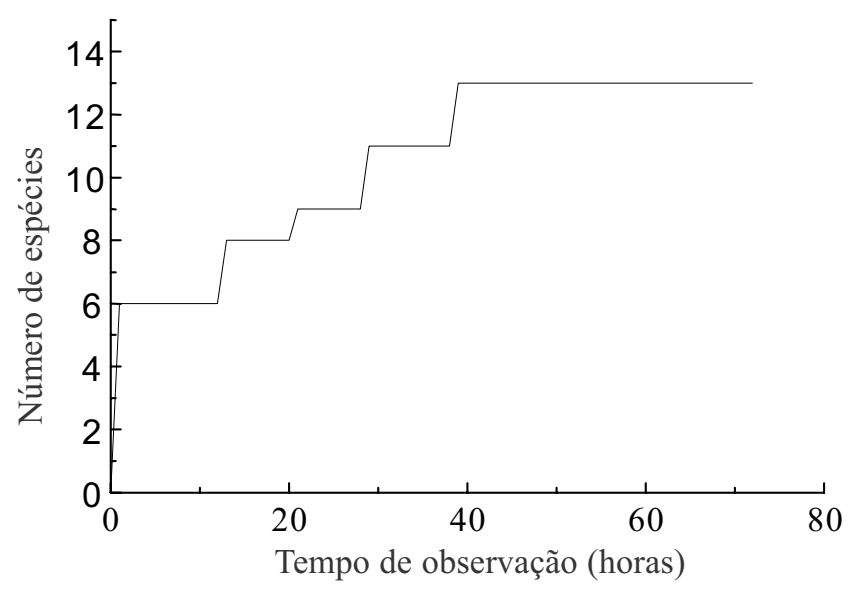

Figura 1. Curva do número cumulativo de espécies de aves observadas visitando Ocotea pulchella em relação ao tempo de observação.

O comportamento de deixar a planta carregando frutos no bico foi observado para Tyrannus melancholichus $(\mathrm{n}=4)$, P. sulphuratus $(\mathrm{n}=4), T$. savana $(\mathrm{n}=1)$, Myiarchus tyrannulus $(\mathrm{n}=1)$ e para Elaenia spp. $(\mathrm{n}=2)$.

M. tyrannulus, P. sulphuratus, $T$. savana e $T$. melancholicus foram observados regurgitando sementes intactas sob as plantas. Um indivíduo de T. leucomelas e um de Turdus amaurochalinus mantidos em cativeiro também regurgitaram as sementes após terem consumido os frutos. Já um exemplar de Schistochlamys ruficapillus, também mantido em cativeiro, tanto regurgitou como defecou sementes inteiras. Embora o tratamento dado às sementes não tenha sido observado diretamente para as outras espécies, provavelmente estas também regurgitem as sementes, pois não foram encontradas fezes com sementes de $O$. pulchella sob as plantas.

Elaenia spp. deixou cair sob as plantas 27,8\% dos frutos capturados antes de serem engolidos $(\mathrm{n}=18), T$. rufiventris $11,6 \%(\mathrm{n}=43)$, T. savana $7,7 \%(\mathrm{n}=39) \mathrm{e}$ M. saturninus 3,6\% $(\mathrm{n}=55)$. Embora $S$. ruficapillus tenha agido na maioria das vezes como dispersor, este foi observado mandibulando $10,3 \%(n=29)$ dos frutos consumidos para a remoção da polpa, deixando a semente cair sob a planta (tabela 2).

Não foi encontrada variação significativa no número de visitas entre os diferentes intervalos de hora quando todas as espécies de aves foram analisadas em conjunto 
(figura 2) e quando T. leucomelas, M. saturninus, Elaenia spp., P. sulphuratus, T. melancholicus e $T$. savana foram analisados separadamente (figura 3). Não houve variação significativa também no tempo de permanência sobre as plantas entre as diferentes espécies de aves (teste de Kruskal-Wallis; kw = 18,09; $\mathrm{p}=0,053)$.

O número de frutos consumidos por visita diferiu significativamente entre as espécies de aves $(\mathrm{kw}=20,19$; $p=0,027)$, tendo sido encontrada correlação significativa

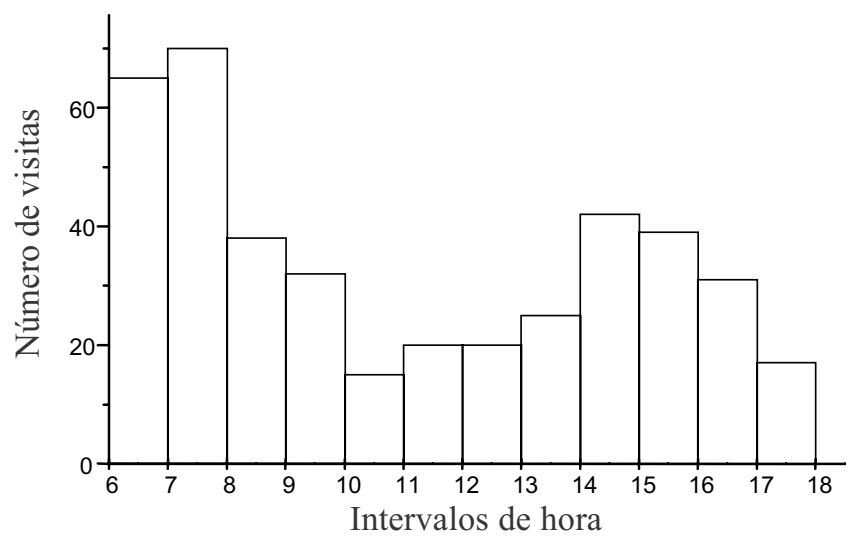

Figura 2. Freqüência de visitas por aves a Ocotea pulchella em diferentes intervalos de hora do dia (teste de KruskalWallis: $\mathrm{kw}=19,23 ; \mathrm{p}=0,057)$. entre o peso das diferentes espécies e o número médio de frutos consumidos $\left(r_{s}=0.89 ; \mathrm{p}<0,001 ; \mathrm{n}=11\right)$.

$\mathrm{O}$ tempo de permanência sobre a planta e o número de frutos consumidos foi positivamente correlacionado para Elaenia spp. $\left(\mathrm{r}_{\mathrm{s}}=0,77 ; \mathrm{p}=0,01 ; \mathrm{n}=10\right)$, M. tyrannulus $\left(\mathrm{r}_{\mathrm{s}}=0,70 ; \mathrm{p}=0,036 ; \mathrm{N}=9\right)$ e T. rufiventris $\left(\mathrm{r}_{\mathrm{s}}=0,97\right.$; $\mathrm{p}=0,016 ; \mathrm{n}=5$ ). Para as demais espécies não foram encontradas correlações significativas: $T$. leucomelas $\left(\mathrm{r}_{\mathrm{s}}=0,34 ; \mathrm{p}=0,33 ; \mathrm{n}=10\right)$, M. saturninus $\left(\mathrm{r}_{\mathrm{s}}=0,37\right.$; $\mathrm{p}=0,27 ; \mathrm{n}=10)$, P. sulphuratus $\left(\mathrm{r}_{\mathrm{s}}=0,13 ; \mathrm{p}=0,71 ; \mathrm{n}=11\right)$, T. amaurochalinus $\left(\mathrm{r}_{\mathrm{s}}=0,31 ; \mathrm{p}=0,49 ; \mathrm{n}=7\right), T$. melancholicus $\left(\mathrm{r}_{\mathrm{s}}=0,57 ; \mathrm{p}=0,57 ; \mathrm{n}=8\right)$, S. ruficapillus $\left(r_{s}=0,25 ; p=0,45 ; n=11\right)$, T. savana $\left(r_{s}=-0,27 ; p=0,44 ;\right.$ $\mathrm{n}=10)$ e Myiozetetes similis $\left(\mathrm{r}_{\mathrm{s}}=-0,20 ; \mathrm{p}=0,91 ; \mathrm{n}=4\right)$.

Os encontros agonísticos intraespecíficos $(\mathrm{n}=18)$ predominaram sobre os encontros interespecíficos $(\mathrm{n}=12)$, sendo observados para $M$. saturninus $(\mathrm{n}=6)$, Elaenia spp. $(\mathrm{n}=4), T$. savana $(\mathrm{n}=3)$, T. leucomelas $(\mathrm{n}=3)$, P. sulphuratus $(\mathrm{n}=1)$ e T. rufiventris $(\mathrm{n}=1)$. Dentre os encontros interespecíficos, M. saturninus foi a principal espécie agressora, tendo interagido com $T$. leucomelas $(\mathrm{n}=5)$, M. tyrannulus $(\mathrm{n}=2), P$. sulphuratus $(\mathrm{n}=1)$ e T. melancholicus $(\mathrm{n}=1)$. As demais interações interespecíficas foram entre $T$. rufiventris e $M$. similis $(\mathrm{n}=1)$, $P$. sulphuratus e $T$. melancholicus $(\mathrm{n}=1)$ e T. leucomelas e T. melancholicus $(\mathrm{n}=1)$.
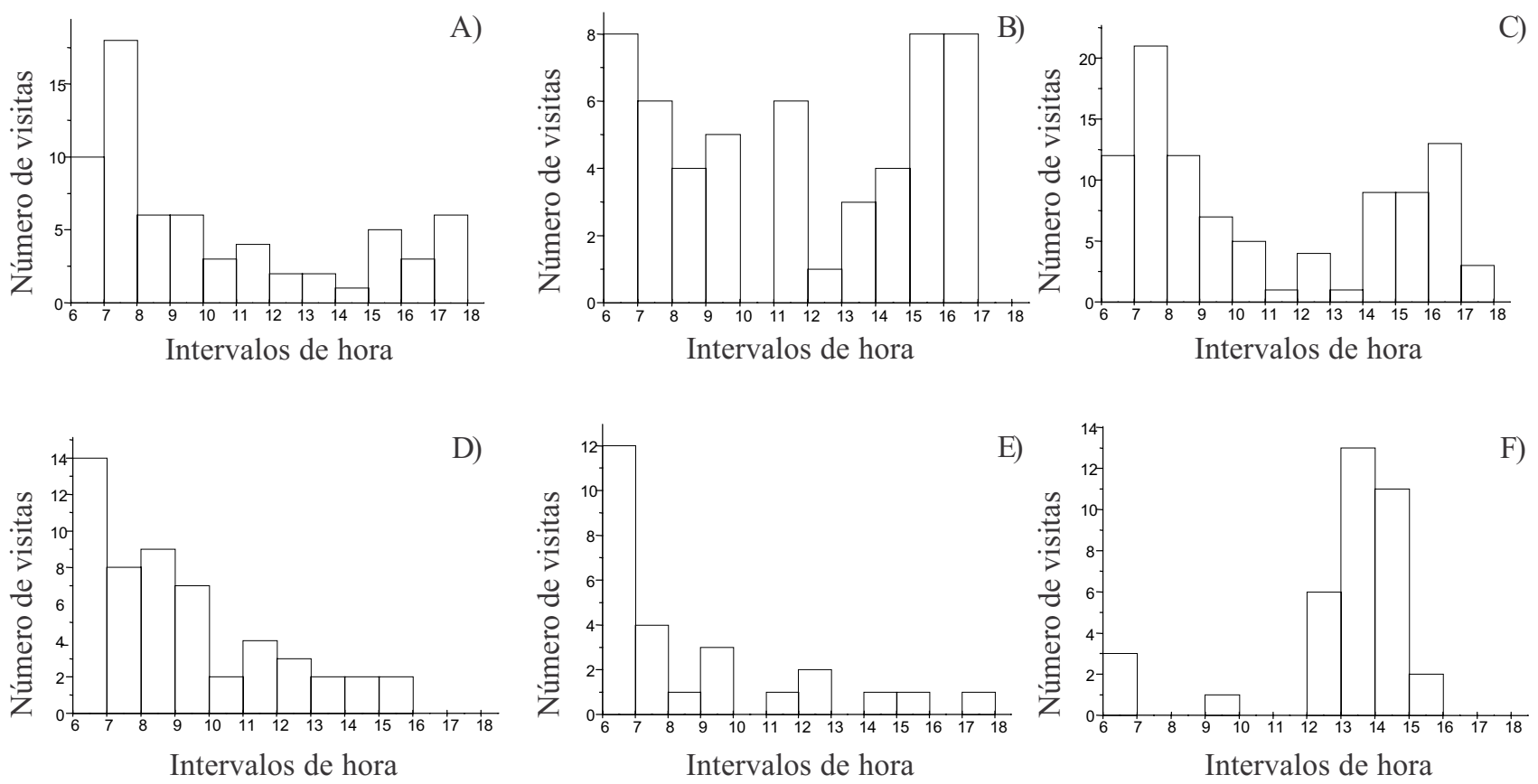

Figura 3. Freqüência de visitas a Ocotea pulchella pelas diferentes espécies de aves em diferentes intervalos de hora do dia. $\mathrm{A}=$ Turdus leucomelas $(\mathrm{kw}=17,32 ; \mathrm{p}=0,09) ; \mathrm{B}=$ Mimus saturninus $(\mathrm{kw}=8,32 ; \mathrm{p}=0,74) ; \mathrm{C}=$ Elaenia $\mathrm{spp} .(\mathrm{kw}=13,33 ; \mathrm{p}=0,27)$; $\mathrm{D}=$ Pitangus sulphuratus $(\mathrm{kw}=15,23 ; \mathrm{p}=0,17) ; \mathrm{E}=$ Tyrannus melancholicus $(\mathrm{kw}=10,96 ; \mathrm{p}=0,44) ; \mathrm{F}=$ T. savana $(\mathrm{kw}=9,36 ; \mathrm{p}=0,59)$. 


\section{Discussão}

Ocotea pulchella demonstrou ser uma espécie generalista quanto ao processo de dispersão, produzindo grandes quantidades de frutos relativamente pequenos que atraíram uma ampla variedade de espécies de aves, tanto onívoras como insetívoras, muitas das quais empregam frutos em suas dietas apenas ocasionalmente. O grande número de visitas sugere que seus frutos constituam um importante recurso alimentar para os dispersores.

Tais características são comumente apresentadas por espécies de plantas de sucessão secundária (Snow 1976), as quais produzem grandes quantidades de pequenas sementes como uma estratégia para colonizarem as áreas abertas disponíveis (Howe 1993). Somando-se a isto, a presença de indivíduos jovens de O. pulchella colonizando áreas alteradas e abertas, como o sub-bosque de um eucaliptal e um cerrado em recuperação, adjacentes à área em estudo (obs. pess.), sugere que esta seja uma espécie de sucessão secundária.

Como já demonstrado por Levey (1987), as aves da subfamília Thraupinae (Emberizidae) podem agir como dispersoras de pequenos frutos cujas sementes não podem ser facilmente removidas da polpa. No entanto, mandibulam frutos geralmente maiores do que $4 \mathrm{~mm}$, deixando as sementes caírem sob as próprias plantas. Corroborando com tal observação, $S$. ruficapillus, embora não tenha agido como dispersor dos propágulos de Copaifera langsdorffii (Caesalpiniaceae) (Motta-Junior \& Lombardi 1990) e Didymopanax macrocarpum (Araliaceae) (M.R. Francisco \& M. Galetti, dados não publicados), cujas larguras são 9,6 e 7,86 mm, respectivamente, se apresentou como um potencial dispersor para $O$. pulchella, cujos frutos são menores.

Embora algumas espécies de aves tenham deixado cair boa parte dos frutos capturados, estas podem ter disponibilizado tais frutos para dispersores secundários, como as formigas saúvas (Atta sp.), que foram observadas removendo frutos caídos sob as plantas. Isto sugere que experimentos futuros possam ser realizados enfocando as formigas como potenciais dispersoras.

Trinta e seis das $223(16,14 \%)$ espécies de aves registradas para a área de estudo são migratórias, permanecendo entre os meses de setembro e abril. Embora a participação de espécies migratórias na dispersão de $O$. pulchella não tenha sido alta, a frutificação dentro deste período aumentou o número de dispersores potenciais (ver Pizo 1997).
Diferentes espécies de aves podem permanecer sobre as plantas por períodos suficientes apenas para se alimentarem, ou podem prolongar os períodos das visitas, de maneira que as sementes dos frutos consumidos passem pelo trato digestivo e sejam eliminadas sob as próprias plantas (Pratt \& Stiles 1983). A inexistência de correlação entre o tempo de permanência sobre as plantas e o número de frutos consumidos para T. leucomelas, M. saturninus, $P$. sulphuratus, T. amurochalinus, T. melancholicus, $S$. ruficapillus, T. savana e $M$. similis pode sugerir que estas espécies tenham prolongado suas visitas, aumentando a probabilidade das sementes serem eliminadas sob as próprias plantas.

As informações quanto à variação do número de visitas às plantas em diferentes horários do dia podem contribuir com a escolha de períodos adequados para a realização de observações, além de revelar os períodos de atividades de frugivoria das diferentes espécies de dispersores. Variações no número de visitas ao longo do dia para espécies de aves foram registradas por Howe (1977) e Kantak (1981). Embora tenham ocorrido encontros agonísticos entre as aves que constituem a guilda de espécies consumidoras dos frutos de $O$. pulchella, as visitas uniformemente distribuídas ao longo do dia sugerem a inexistência de exclusão competitiva temporal entre elas (ver Galetti et al. 1999).

Tais encontros agonísticos, inter e intraespecíficos, podem constituir um fator determinante para a dispersão. Em trabalhos anteriores, T. leucomelas foi considerada uma das espécies de maior agressividade $(63 \%$ das investidas; $n=68$ ), permanecendo por longos períodos sobre a copa impedindo a aproximação de outras aves potencialmente dispersoras, o que é um fator negativo para a dispersão (Pizo 1997). Para O. pulchella, M. saturninus foi a principal espécie agressora (50\% das investidas; $\mathrm{n}=30$ ). No entanto, o número de investidas foi bastante baixo em relação ao número total de visitas, sugerindo que estes encontros não tenham interferido de maneira significativa no processo de dispersão.

As teorias que tratam da dispersão de sementes por aves frugívoras têm dado ênfase à alta qualidade da dispersão desempenhada por aves exclusivamente frugívoras, em contraposição àquelas que também exploram outros recursos, como insetos (Pratt \& Stiles 1983). Por outro lado, alguns estudos têm demonstrado que dispersores frugívoros especialistas nem sempre garantem uma dispersão mais eficiente em relação aos dispersores generalistas (Wheelwright \& Orians 1982, Moermond \& Denslow 1985). Embora espécies primariamente frugívoras não tenham sido observadas 
consumindo os frutos de $O$. pulchella, as espécies oportunistas garantiram altas freqüências de visitas, altas taxas de consumo e permaneceram por curtos períodos de tempo sobre as plantas, sugerindo que estejam desenvolvendo um papel importante na dispersão. Além disso, as espécies dispersoras aqui observadas são bastante comuns e muito freqüentes inclusive em áreas antrópicas, demonstrando a capacidade de plantas secundárias e generalistas, como $O$. pulchella, de se estabelecerem mesmo em ambientes degradados.

Agradecimentos - Os autores são gratos à Profa. Dra. Maria Inês Salgueiro Lima, do Departamento de Botânica da Universidade Federal de São Carlos, pela identificação da planta em estudo. M. Galetti recebe financiamento do $\mathrm{CNPq}$ (300025/97-1) e FAPESP (96/10464-7) e M. R. Francisco recebe financiamento da CAPES.

\section{Referências bibliográficas}

BLAKE, J.G., LOISELLE, B.A., MOERMOND, T.C., LEVEY, D.J. \& DENSLOW, J.S. 1990. Quantifying abundance of fruits for birds in tropical habitats. Studies in Avian Biology 13:73-79.

COATES-ESTRADA, R. \& ESTRADA, A. 1988. Frugivory and seed dispersal in Cymbopetalum baillonii (Annonaceae) at Los Tuxtlas, Mexico. Journal of Tropical Ecology 4:157-172.

FOSTER, M.S. 1987. Feeding methods and efficiencies of selected frugivorous birds. The Condor 89:566-580.

GALETTI, M. \& PIZO, M.A. 1996. Fruit eating birds in a forest fragment in southeastern Brazil. Ararajuba 4:71-79.

GALETTI, M. \& STOTZ, D. 1996. Miconia hypoleuca (Melastomataceae) como espécie-chave para aves frugívoras no sudeste do Brasil. Revista Brasileira de Biologia 56:435-439.

GALETTI, M., ZIPPARRO, V. \& MORELLATO, L.P. 1999. Fruit phenology and frugivory on the palm Euterpe edulis in a lowland Atlantic forest. Ecotropica 5:115-122.

HERRERA, C.M., JORDANO, P., LÓPEZ-SORIA, L. \& AMAT, J.A. 1994. Recruitment of a mast-fruiting, bird-dispersed tree: bridging frugivore activity and seedling establishment. Ecological Monographs 64:315-344.

HOWE, H.F. 1977. Bird activity and seed dispersal of a tropical wet forest tree. Ecology 58:539-550.

HOWE, H.F. 1993. Specialized and generalized dispersal systems: where does "the paradigm" stand ? Vegetatio 107/108:3-13.

HOWE, H.F. \& ESTABROOK, G.F. 1977. On intraspecific competition for avian dispersers in tropical trees. The American Naturalist 111:817-832.
HOWE, H.F. \& SMALLWOOD, J. 1982. Ecology of seed dispersal. Annual Review of Ecology and Systematics 13:201-228.

KANTAK, G.E. 1981. Temporal feeding patterns of some tropical frugivores. The Condor 83:185-187.

LEVEY, D.J. 1987. Seed size and fruit-handling techniques of avian frugivores. The American Naturalist 129:471-485.

LORENZI, H. 1998. Árvores brasileiras: manual de identificação e cultivo de plantas arbóreas nativas do Brasil, v. I. Editora Plantarum, Nova Odessa.

MARINI, M.A., MOTTA-JUNIOR, J.C., VASCONCELLOS, L.A.S. \& CAVALCANTI, R.B. 1997. Avian body masses from the cerrado region of central Brazil. Ornitologia Neotropical 8:93-99.

MOERMOND, T.C. \& DENSLOW, J.S. 1985. Neotropical avian frugivores: patterns of behavior, morphology, and nutrition, with consequences for fruit selection. Ornithological Monographs 36:865-897.

MOTTA-JUNIOR, J.C. 1990. Estrutura trófica e composição das avifaunas de três hábitats terrestres na região central do estado de São Paulo. Ararajuba 1:65-71.

MOTTA-JUNIOR, J.C. \& LOMBARDI, J.A. 1990. Aves como agentes dispersores da copaíba (Copaifera langsdorffii, Caesalpiniaceae) em São Carlos, estado de São Paulo. Ararajuba 1:105-106.

MOTTA-JUNIOR, J.C. \& VASCONCELLOS, L.A.S. 1996. Levantamento das aves do campus da Universidade Federal de São Carlos, estado de São Paulo, Brasil. In Anais do VII Seminário Regional de Ecologia. Universidade Federal de São Carlos, São Carlos, p.159-171.

PAESE, A. 1997. Caracterização e análise ambiental do campus da Universidade Federal de São Carlos (UFSCar), São Carlos, SP. Dissertação de mestrado, Universidade Federal de São Carlos, São Carlos.

PIZO, M.A. 1997. Seed dispersal and predation in two populations of Cabralea canjerana (Meliaceae) in the Atlantic Forest of southeastern Brazil. Journal of Tropical Ecology 13:559-578.

PRATT, T.K. \& STILES, E.W. 1983. How long fruit-eating birds stay in the plants where they feed: implications for seed dispersal. The American Naturalist 122:797-805.

SCHUPP, E.W. 1993. Quantity, quality and the effectiveness of seed dispersal by animals. Vegetatio 107/108:15-29.

SICK, H. 1997. Ornitologia Brasileira. Editora Nova Fronteira, Rio de Janeiro.

SNOW, D.W. 1976. The web of adaptation: bird studies in the American tropics. Cornell University Press, New York.

SNOW, D.W. 1981. Tropical frugivorous birds and their food plants: a world survey. Biotropica 13:1-14.

SOKAL, R.R. \& ROHLF, F.J. 1981. Biometry. W.H. Freeman, New York. 